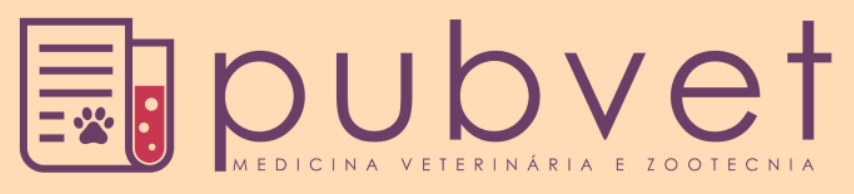

HTTP://DX.DOI.ORG/10.22256/PUBVET.V1IN12.1239-1244

\title{
Osteossarcoma canino: Relato de caso
}

\section{Rebecca Tavares Lima ${ }^{1 *}$, Mariela Sousa Gomes $^{2}$, Veridiana Miranda Negreiros ${ }^{3}$, Leopoldo Fabrício Marçal do Nascimento ${ }^{4}$}

${ }^{\text {I}}$ Especialista em Diagnóstico por Imagem e em Clínica Médica e Cirurgia de Cães e Gatos, Universidade Federal do Piauí, Teresina, Piauí, Brasil-bebektavares@hotmail.com

${ }^{2}$ Especialista em Diagnóstico por Imagem, Universidade Federal do Piauí, Teresina, Piauí, Brasil - mariela.gomes@hotmail.com

${ }^{3}$ Especialista em Clínica Médica e Cirurgia de Cães e Gatos, Instituto Qualittas de Pós-Graduação, Teresina, Piauí veridianamiranda_negreiros@hotmail.com

${ }^{4}$ Mestre em Ciência Animal, Universidade Federal do Piauí, Teresina, Piauí - leopoldofmarcal@gmail.com

*Autor para correspondência

RESUMO. Osteossarcoma é um dos tumores ósseos maligno mais comum em cães, sendo que nessa espécie mais de $80 \%$ das neoplasias ósseas malignas são osteossarcomas. A ocorrência desta doença é maior em cães com 5 a 9 anos, com pico ao redor de 7 anos, porém ela também pode ser observada em cães jovens, com 1 ano de idade. Em raças de grande porte a incidência é maior e ambos os sexos são igualmente acometidos. $\mathrm{O}$ osteossarcoma ocorre também em gatos, mas é menos comum do que em cães, e o desenvolvimento de metástases é mais lento. A etiologia do osteossarcoma canino permanece desconhecida. Os primeiros sintomas a serem observados são claudicação e extenso aumento de volume, doloroso, no foco tumoral. O diagnóstico é baseado no histórico, exame físico, laboratorial, citilográficos e principalmente achados radiográficos, que mostram áreas de destruição óssea, neoformação óssea, contorno indefinido, reação periosteal (em alguns casos em "raios de sol") e aumento do volume de tecidos moles, sendo a confirmação feita por biópsia e exame histopatológico. Os tratamentos podem ser com quimioterapia, radioterapia, cirurgia para a amputação do membro acometido e cirurgia com preservação de membro. O prognóstico em geral é ruim, porém dependerá da espécie, da raça e da resposta ao tratamento.

Palavras chave: Neoplasia óssea, osteossarcoma, tumor ósseo

\section{Canine osteosarcoma: Case report}

ABSTRACT. Osteosarcoma is one of the most common malignant bone tumors in dogs, and in this species more than $80 \%$ of malignant bone neoplasms are osteosarcomas. The occurrence of this disease is greater in dogs with 5 to 9 years, with a peak around 7 years, but it can also be observed in young dogs, at 1 year of age. In large breeds the incidence is higher and both sexes are equally affected. Osteosarcoma also occurs in cats, but is less common than in dogs, and the development of metastases is slower. The etiology of canine osteosarcoma remains unknown. The first symptoms to be observed are lameness and extensive painful volume increase in the tumor focus. The diagnosis is based on the history, physical examination, laboratory examination, scintigraphy and mainly radiographic findings, showing areas of bone destruction, bone neoformation, indefinite contour, periosteal reaction (in some cases in "sun rays") and increase in tissue volume The biopsy and histopathological examination were confirmed. The treatments can be with chemotherapy, radiotherapy, surgery for amputation of the affected limb and surgery with limb preservation. The prognosis is usually poor, but will depend on the species, the breed and the response to treatment.

Key words: Bone neoplasia, osteosarcoma, bone tumor 


\section{Osteosarcoma canino: Reporte de un caso}

RESUMEN. Osteosarcoma es uno de los tumores óseos malignos más común en perros, siendo que en esa especie más del $80 \%$ de las neoplasias óseas malignas son osteosarcomas. La ocurrencia de esta enfermedad es mayor en perros de 5 a 9 años, con pico alrededor de 7 años, pero también se puede observar en perros jóvenes, con 1 año de edad. En razas de gran tamaño la incidencia es mayor y ambos sexos son igualmente acometidos. El osteosarcoma se presenta también en gatos, pero es menos común que en perros, y el desarrollo de metástasis es más lento. La etiología del osteosarcoma canino sigue siendo desconocida. Los primeros síntomas a ser observados son claudicación y extenso aumento de volumen, doloroso, en el foco tumoral. El diagnóstico se basa en el historial, examen físico, examen de laboratorio, examen citilográfico y principalmente hallazgos radiográficos, que muestran áreas de destrucción ósea, neoformación ósea, contorno indefinido, reacción periosteal (en algunos casos en "rayos de sol") y aumento del volumen de tejidos blandos, siendo la confirmación hecha por biopsia y examen histopatológico. Los tratamientos pueden ser con quimioterapia, radioterapia, cirugía para la amputación del miembro acometido y cirugía con preservación de miembro. El pronóstico en general es reservado, pero dependerá de la especie, de la raza y de la respuesta al tratamiento.

Palabras clave: Neoplasia ósea, osteosarcoma, tumor óseo

\section{Introdução}

As neoplasias ósseas primárias representam de 3 a $4 \%$ dos tumores malignos em animais da espécie canina, sendo também descrita a sua ocorrência em felinos e humanos (Daleck et al., $\underline{2002}$, Boerman et al., 2012, Wolfesberger et al., 2016).

O osteossarcoma (OSA) é uma neoplasia óssea, maligna, correspondendo a aproximadamente $85 \%$ dos tumores ósseos primários nos cães (Sabattini et al., 2017). Em geral essa enfermidade ocorre com maior frequência em cães de raças grandes ou gigantes, com idade média de sete anos e meio e em região metafisária dos ossos (Anderson, 1996).

Os locais de predileção do OSA são as regiões dos ossos longos (úmero, fêmur, raio, tíbia e ulna) (Liptak et al., 2004) com aproximadamente 25\% dos tumores que surgem no esqueleto axial, incluindo os ossos planos do crânio, costelas, vértebras, esterno e pélvis (Hammer et al., 1995, Dickerson et al., 2001).

Por tratar-se de um tumor agressivo, a presença de metástase pode ser evidenciada precocemente no curso da doença, sendo a causa mais comum de morte do paciente, rapidamente formadas e primariamente pulmonares nos cães (Spodnick (Spodnick et al., 1992, Anderson, 1996, Berg, 1996). Em torno de 90\% das lesões metastáticas são encontradas nos pulmões, sendo os $10 \%$ restantes localizados em outros órgãos ou em outros ossos (Newton et al., 1992).
A amputação é o procedimento cirúrgico mais comum recomendado para OSA para remover simultaneamente o tecido neoplásico e aliviar a dor, assegurando baixa morbidade em termos de deiscência e infecção (Spodnick et al., 1992).

O objetivo deste trabalho foi relatar um caso de osteossarcoma canino com presença de metástase e achados necroscópicos e laboratoriais característicos da doença.

\section{Relato de caso}

No presente estudo, relata-se o caso de um cão da raça Rottweiller, fêmea, com seis anos e nove meses de idade, $35,4 \mathrm{~kg}$, atendido no Hospital Veterinário Universitário da Universidade Federal do Piauí (HVU/UFPI). O proprietário relatava um histórico de claudicação em membro pélvico esquerdo com evolução em três semanas (Figura 1). Na anamnese, foi relatado que o paciente teve quadros de vômitos, passou a defecar pastoso e mais escuro, comendo pouco e aumentando a ingestão de água. Não havia histórico de queda ou qualquer outro traumatismo. $\mathrm{O}$ animal possuía vacinação em dia, tinha histórico de presença de ectoparasitas (carrapatos) e fazia o controle por meio de medicamento injetável.

Ao exame clinico, presenciou-se aumento de volume e dor do membro acometido acompanhado de linfadenopatia generalizada, foi constatado bom estado nutricional e de hidratação, temperatura retal de $39,1^{\circ} \mathrm{C}$, frequência cárdica de 160 batimentos por minuto, frequência respiratória de 30 movimentos por minuto e 
ausculta cardiopulmonar sem alteração aparente. As mucosas apresentavam-se discretamente hipocoradas, o tempo de preenchimento capilar inferior a 2 segundos e palpação abdominal sem alterações.

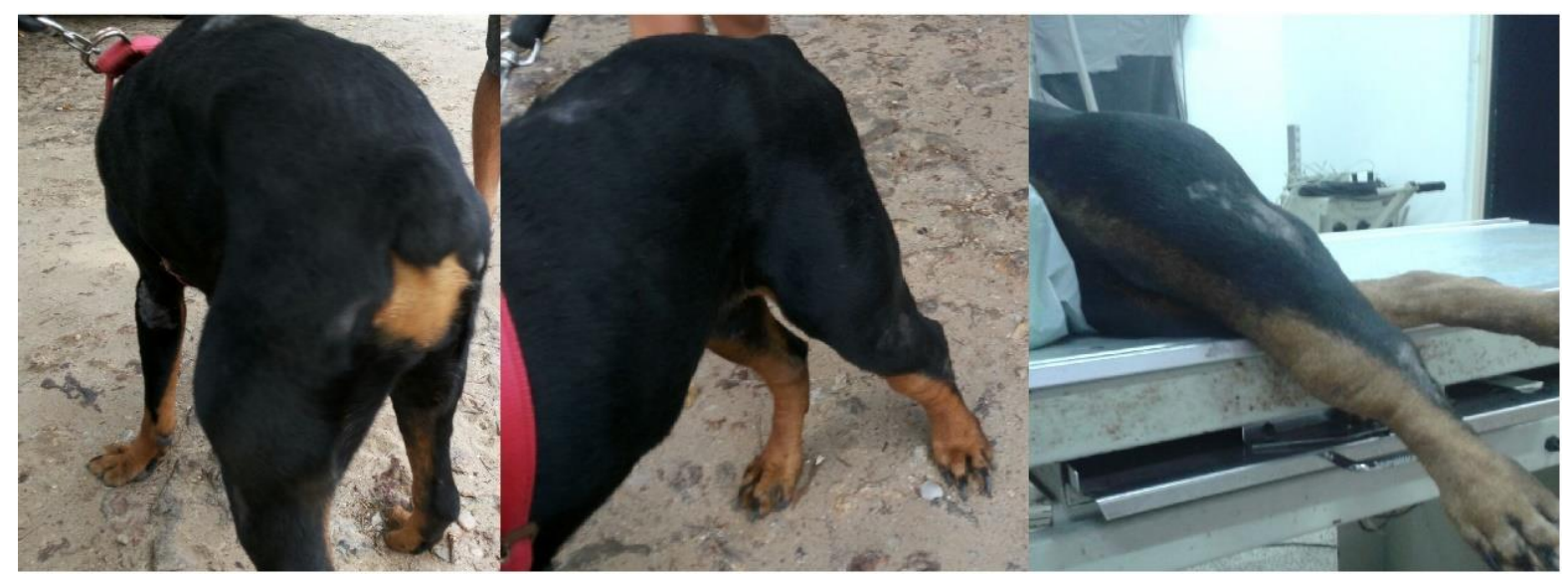

Figura.1 Aumento de volume do membro pélvico esquerdo

Com base na sintomatologia clínica, foram realizados exames de radiografia de fêmur esquerdo na posição médio lateral/crânio caudal, radiografia de tórax na posição latero lateral e ventro dorsal, hemograma, dosagem de creatinina, alaninotransferase (ALT), fosfatase alcalina, proteína total, albumina, globulina, cálcio e fósforo.

\section{Resultados e Discussão}

A análise hematológica mostrou anemia normocitica normocromia, trombocitose e leucocitose com neutrófila, além da observação de estruturas sugestivas de mórula de Anaplasma platys. $\mathrm{Na}$ análise bioquímica foi encontrado aumento da fosfatase alcalina, hipoproteinemia, hipoalbuminemia, seguido de hipocalcemia.

As radiografias de fêmur revelaram áreas de intensa lise óssea associadas a áreas de proliferação de erosão no córtex se estendendo para o interior dos tecidos moles circunjacentes em região distal de fêmur esquerdo (Figura 2).

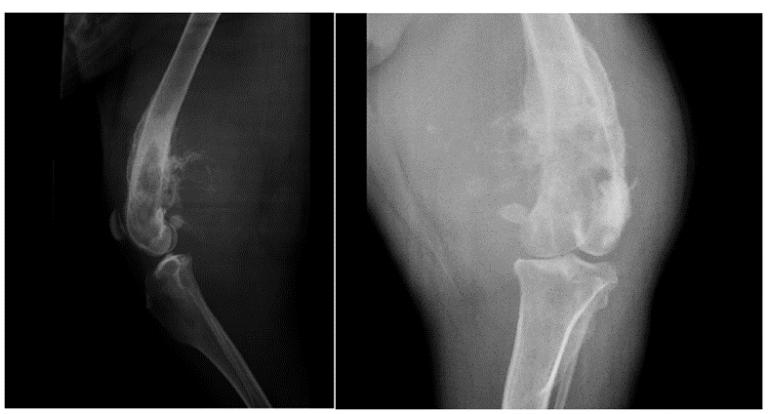

Figura.2 Radiografia de fêmur esquerdo na posição médio lateral/crânio caudal.
Nas radiografias torácicas observaram-se padrão pulmonar misto intersticial e alvéolos com presença de nódulos circunscritos radiopacos distribuídos aleatoriamente no campo pulmonar sugestivos de metástase (Figura 3). Sugeriu-se ao proprietário, procedimento cirúrgico de amputação do membro e terapia de cisplatina, mas proprietário recusou-se optando pela eutanásia do animal.

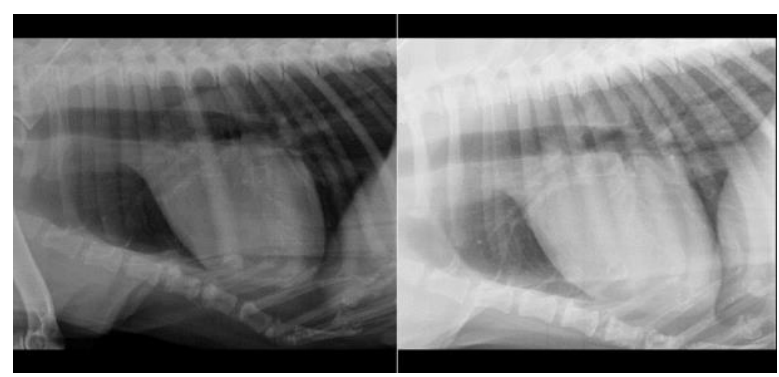

Figura 3. Radiografia de tórax na posição latero lateral direta esquerda e esquerda direita

A necropsia foi realizada e verificou-se carcaça e mucosas discretamente pálidas. Edema subcutâneo, hemorragia de membro posterior esquerdo com presença de aumento de volume com superfície friável (Figura 4A).

A abertura da cavidade torácica observou-se moderada quantidade de liquido espumoso de coloração avermelhada. Traqueia com presença de liquido espumoso de coloração avermelhada. Pulmões com pontos firmes brancacentos, mais pesados que o normal, superfície brilhante, fluindo liquido à abertura dos brônquios (Figura 4B). Na abertura do saco pericárdio foi observada grande quantidade de liquido avermelhado, coração com presença de espessamento nodular de coloração 
brancacenta, consistência firme, localizada na válvula atrioventricular esquerda. A abertura da cavidade abdominal observou-se moderada quantidade de liquido de coloração avermelhada. Fígado com lobulação evidente, aumentado de volume, com bordas arredondadas de coloração amarelada, friável e de aspecto untuoso. Baço com pontos brancacentos que variam de 0,3 a $0,5 \mathrm{~mm}$. Os linfonodos foram também avaliados, porém não foram detectadas células tumorais.

$\mathrm{Na}$ oncologia veterinária, o OSA é definido como um tumor mesenquial primário maligno cujas células cancerosas produzem matriz osteoide, sendo um tumor ósseo que ocorre frequentemente em cães, afetando principalmente animais de grande porte, meia idade (Kleiner and Silva, 2003) e possuindo maior risco de incidência em cães com peso acima de $36 \mathrm{~kg}$ (Kleiner and Silva, 2003, Soares et al., 2005), o que confirma os resultados encontrados neste animal.

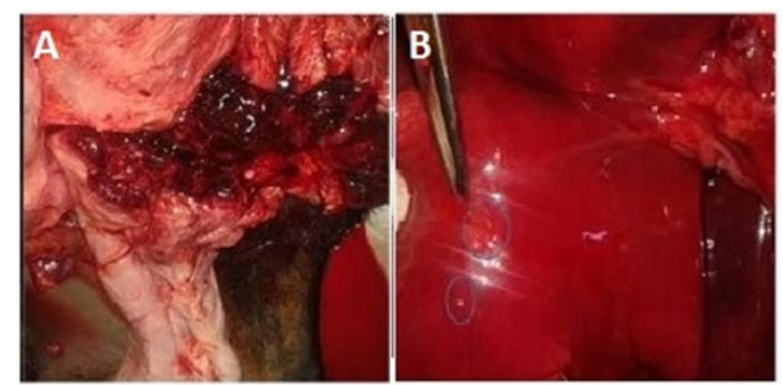

Figura 4. A - Edema subcutâneo, hemorragia de membro pélvico esquerdo com presença de aumento de volume com superfície friável; B - Pulmões com pontos firmes brancacentos.

O OSA faz parte de um grupo heterogêneo de neoplasias malignas que afetam a formação de osso ou tecido mesenquimal, sendo caracterizado pela diversidade de aspectos histopatológicos e de comportamento clinico biológico (Soares et al., 2005, Martelli et al., 2007), muito agressivo e com um crescimento rápido (Bastos et al., 1999), altamente metastático e com grande predileção pelo pulmão, em torno de $90 \%$ dos casos (Newton et al., 1992).

Cães com tumores ósseos apendiculares apresentam dor e claudicação no sitio do tumor primário. A dor é devida microfraturas ou a interrupção do periósteo induzidos pela osteólise do osso cortical pela extensão tumoral do canal medular. $\mathrm{O}$ animal pode apresentar ainda tumefação na extremidade proximal do fêmur, tíbia e úmero (Dernell et al., 2001).

Os cães machos são diagnosticados com osteossarcoma 1,1 a 2 vezes mais frequentes que as fêmeas (Endicott, 2003). No Brasil não se tem uma estatística a respeito das principais raças acometidas, suas respectivas faixas etárias e localização do osteossarcoma. No entanto, alguns relatos publicados no Brasil, demonstram que, cães portadores desta neoplasia são de grande porte, sendo as principais raças afetadas: Fila Brasileiro, Doberman, Rotweiller e Pastor Alemão. Segundo os mesmos estudos, as maiores incidências, nestes casos foram nos ossos úmero e tíbia (Daleck et al., 2002).

O reconhecimento clínico desta neoplasia com localização axial é mais difícil que aquele do esqueleto apendicular. Os sinais variam desde edema localizado com ou sem claudicação a disfagia (sítio oral), exoftalmia e dor ao abrir a boca (sitio orbital ou mandíbula caudal), deformidade facial, descargas nasais, espirros (sitio em cavidade e seios nasais) e hiperestesia com ou sem sinais neurológicos (medula espinhal) (Dernell et al., 2001).

A dosagem da enzima fosfatase alcalina no soro pode ser considerada uma ferramenta valiosa no prognóstico, por ser um marcador de renovação óssea (Boerman et al., 2012). Quando se apresenta elevada no momento do diagnóstico do tumor ou após o tratamento primário do tumor, pode indicar evidencia clínica de metástases (Daleck et al., 2002, Sternberg et al., 2013), sendo assim observado no animal em questão, onde ao se avaliar a fosfatase alcalina elevada, pôde se estabelecer a conexão com a metástase pulmonar.

Estabelecido o diagnóstico quando não há sinais de metástase, a amputação do membro ainda é o tratamento de escolha de cães com osteossarcoma (Nelson and Couto, 2015). O tempo médio de sobrevida de cães com osteossarcoma apendicular tratados apenas com a amputação é de aproximadamente quatro meses (Spodnick et al., 1992, Nelson and Couto, 2015), ao passo que aqueles cães tratados com a amputação mais cisplatina apresentam sobrevida média significativamente mais prolongada que os cães tratados apenas com amputação (Murphy et al., 2017).

Novas abordagens terapêuticas para cães com osteossarcoma radiais vêm sendo utilizada (Nelson and Couto, 2015). Esta técnica é a preservação da função do membro chamado de "Limb Sparing", que consiste na exérese do segmento ósseo envolvido com a neoplasia, com uma margem de segurança de aproximadamente 2 $\mathrm{cm}$. A seguir faz-se o implante de uma prótese 
óssea conservada a baixa temperatura e fixada com uma placa metálica. Associada a cirurgia, utiliza-se à cisplatina como agente quimioterápico (Daleck et al., 2002, Steffey et al., 2017).

\section{Conclusão}

O osteossarcoma é uma neoplasia maligna de prognóstico ruim. Os métodos de diagnóstico implicados para esta afecção apresentam, muitas vezes, carências principalmente em relação ao tempo estimado para o estabelecimento do mesmo. Sendo assim, a terapia é quase sempre iniciada em estágios mais avançados, quando o animal desenvolve a clínica, dificultando o tratamento dos cães especialmente nos casos com metástase e, diminuindo a expectativa do proprietário. A terapia não leva a cura do animal, mas busca uma melhoria na qualidade de vida do mesmo, principalmente em relação ao desconforto originado pelo tumor. $\mathrm{O}$ procedimento cirúrgico associado a outras terapias adjuvantes, como a quimioterapia, parece ser ainda o mais indicado a estes pacientes.

\section{Referências Bibliográficas}

Anderson, T. J. 1996. Sarcomas óseos primarios del perro y del gato œ diagnóstico y opciones de tratamiento. Walthan Focus, 6, 21-30.

Bastos, T. M. M., Serafini, O. A., Barrios, C. H. E. \& Velasco, P. A. 1999. Osteossarcoma: tratamento e fatores progn $\varnothing$ sticos. Revista Brasileira de Ortopedia, 34, 59-62.

Berg, J. 1996. Canine osteosarcoma: amputation and chemotherapy. Veterinary Clinics of North America: Small Animal Practice, 26, 111-121.

Boerman, I., Selvarajah, G. T., Nielen, M. \& Kirpensteijn, J. 2012. Prognostic factors in canine appendicular osteosarcoma-a metaanalysis. BMC Veterinary Research, 8, 8-56.

Daleck, C. R., Fonseca, C. S. \& Canola, J. C. 2002. Osteossarcoma canino-revisão. Revista de Educação Continuada em Medicina Veterinária e Zootecnia, 5, 233-242.

Dernell, W. S., Ehrhart, N. P., Straw, R. C. \& Vail, D. M. 2001. Tumors of the skeletal system. Small Animal Clinical Oncology, 3, 378-417.

Dickerson, M. E., Page, R. L., LaDue, T. A., Hauck, M. L., Thrall, D. E., Stebbins, M. E. \& Price, G. S. 2001. Retrospective analysis of axial skeleton osteosarcoma in 22 large-breed dogs. Journal of Veterinary Internal Medicine, $15,120-124$.
Endicott, M. 2003. Principles of treatment for osteosarcoma. Clinical Techniques in Small Animal Practice, 18, 110-114.

Hammer, A. S., Weeren, F. R., Weisbrode, S. E. \& Padgett, S. L. 1995. Prognostic factors in dogs with osteosarcomas of the flat or irregular bones. Journal of the American Animal Hospital Association, 31, 321-326.

Kleiner, J. A. \& Silva, E. G. 2003. Bone tumors affecting small animals. Revista Científica de Medicina Veterinária, 1, 193-200.

Liptak, J. M., Dernell, W. S., Straw, R. C., Rizzo, S. A., Lafferty, M. H. \& Withrow, S. J. 2004. Proximal radial and distal humeral osteosarcoma in 12 dogs. Journal of the American Animal Hospital Association, 40, 461-467.

Martelli, A., Teixeira, L. B. C. \& Santos Júnior, A. R. 2007. Aspectos histopatológicos e histoquimico de osteossarcomas em caes. Estud Biol, 29, 179-89.

Murphy, B. G., Mok, M. Y., York, D., Rebhun, R., Woolard, K. D., Hillman, C., Dickinson, P. \& Skorupski, K. 2017. Evaluation of P16 expression in canine appendicular osteosarcoma. BMC Veterinary Research, 13, 189.

Nelson, R. W. \& Couto, C. G. 2015. Medicina interna de pequenos animais. Elsevier Editora, Amsterdan.

Newton, C. D., Biery, D. N. \& Ettinger, S. J. 1992. Moléstias esqueléticas. In: Ettinger, S. J. (ed.) Tratado de Medicina Interna Veterinária. Manole, São Paulo.

Sabattini, S., Renzi, A., Buracco, P., Defourny, S., Garnier-Moiroux, M., Capitani, O. \& Bettini, G. 2017. Comparative Assessment of the Accuracy of Cytological and Histologic Biopsies in the Diagnosis of Canine Bone Lesions. Journal of Veterinary Internal Medicine, 31, 864-871.

Soares, R. C., Soares, A. F., Souza, L. B., Santos, A. L. V. \& Pinto, L. P. 2005. Osteossarcoma de mandíbula inicialmente mimetizando lesão do periápice dental: relato de caso. Brazilian Journal of Otorhinolaryngology, 71, 242-245.

Spodnick, G. J., Berg, J., Rand, W. M., Schelling, S. H., Couto, G., Harvey, H. J., Henderson, R. A., MacEwen, G., Mauldin, N. \& McCaw, D. L. 1992. Prognosis for dogs with appendicular osteosarcoma treated by amputation alone: 162 
cases (1978-1988). Journal of the American Veterinary Medical Association, 200, 995-999.

Steffey, M. A., Garcia, T. C., Daniel, L., Zwingenberger, A. L. \& Stover, S. M. 2017. Mechanical properties of canine osteosarcomaaffected antebrachia. Veterinary Surgery, 46, 539-548.

Sternberg, R. A., Pondenis, H. C., Yang, X., Mitchell, M. A., O'brien, R. T., Garrett, L. D., Helferich, W. G., Hoffmann, W. E. \& Fan, T. M. 2013. Association between absolute tumor burden and serum bone-specific alkaline phosphatase in canine appendicular osteosarcoma. Journal of Veterinary Internal Medicine, 27, 955-963.
Wolfesberger, B., Fuchs-Baumgartinger, A., Hlavaty, J., Meyer, F. R., Hofer, M., Steinborn, R., Gebhard, C. \& Walter, I. 2016. Stem cell growth factor receptor in canine vs. feline osteosarcomas. Oncology Letters, 12, 24852492.

\section{Article History:}

Received 15 July 2017

Accepted 15 August 2017

Available on line 20 October 2017

License information: This is an open-access article distributed under the terms of the Creative Commons Attribution License 4.0, which permits unrestricted use, distribution, and reproduction in any medium, provided the original work is properly cited. 